\title{
Develop Web GIS Based Intelligent Transportation Application Systems with Web Service Technology
}

\author{
Xiaolin Lu \\ College of Information Technology, Zhejiang University of Finance \& Economics \\ Hangzhou, Zhejiang, China 310018
}

\begin{abstract}
The GIS Web Service technology provides a new mechanism and an effective method to develop next generation GIS system. This paper investigates the method to develop Web GIS based ITS application systems with GIS web service technology. Based on the OGC GIS Web Service framework and SOA architecture, a distributed and web service enabled WebGIS based ITS application system is investigated. The GIS-T Web Services are exposed and discovered in the GIS-T web service portal. Through GIS-T web service, the hosted spatial data and GIS functionalities can be accessed and integrated to the different customized ITS applications. The GIS content and capabilities can be integrated into the customized ITS application systems for sharing geographical information and data. ITS application systems can access GIS-T Web Services through Web protocols such as HTTP and XML without concern for how each service is implemented. The GIS-T web service is a promising architecture for implementation next generation GIS-T information systems. It has very good promotion prospect and important value for the system design and construction for various Web GIS based ITS application systems.
\end{abstract}

\section{INTRODUCTION}

The GIS technologies used for the ITS (Intelligent Transportation System) have been widely studied by many investigators in recent years. With the rapidly development and widely application of ITS, GIS-T (GIS for Transportation) technologies are increasingly urgent demanded. The research and development of GIS based intelligent transportation application systems have become a more and more important research area in ITS. GIS mapping for the intelligent transportations system is an essential, efficient, and effective tools for planning and managing transportation information resources $[1,2]$.

With the development of the Internet, the traditional GIS based ITS application systems are needed to transform to Web enable ITS system for widely accessible and convenient usable in browser. However, the development of the Web GIS based ITS application systems requires the customer to increase more effort and repetition works for constructing the Web GIS system for the different ITS systems. As the technology of web services evolves, the web service becomes a main working pattern and a significant application model for next generation Internet application [3,4]. The GIS web services can provide the hosted spatial data and GIS functionality to integrate the customized ITS applications to perform basic geo-processing tasks, such as address matching, map image display, and routing, without maintaining GIS tools or the associated geographical data. Therefore, the GIS Web service technology is very promising for developing the next generation Web GIS based ITS applications systems [5].

In this paper, through the analysis of the framework and GIS Web Services technology and system architecture, we investigate and analysis the service-oriented architecture for building a distributed and web service enabled Web-GIS based intelligent transportation application system. We designed and implemented a prototype of the service enabled Web-GIS intelligent transportation application system.

\section{OVERVIEW OF RELATED WORKS}

With the development of the Internet, the GIS information systems become more and more used by large-scale area and public society [6,7]. The traditional GIS ITS application systems are urgent need transform the Web based systems. The emerging Web Services technology is the software development revolution and the revolution for the next generation Internet, which provided a new mechanism and an effective method to solve the problem of software systems integration and data sharing. Web Services are the software components that are well defined, self-contained, and does not depend on the context or state of other services. Web services essentially use XML to create a robust connection.

There are a number of researches and developing projects of applying the web services to GIS system by several different companies and organizations. The Environmental Systems Research Institute (ESRI) reported the ArcWeb Services that could include and integrate the GIS content and capabilities in the customized applications for sharing geographical information and data [8]. Applications can access Web Services through Web protocols such as HTTP and XML, without concern for how each service is implemented. Microsoft developed the MapPoint.NET Web Service for GIS mapping and location services that could be used for developing a broad range of location enhanced applications, including wireless/mobility, customer relationship management, enterprise location services, and business intelligence [9]. Microsoft MapPoint .NET is a hosted, programmable XML Web Service that allows application developer to integrate high-quality maps, driving directions, distance calculations, and proximity searches into their applications, business processes, and web sites.

The Open GIS Consortium (OGC) developed the web-based geographic information and services accessible via the Internet through the OGC consensus process $[10,11]$. Each service 
supports a certain GIS operation function that returns metadata describing the nature of the service, the methods to register Web Service, the addresses to access the service or to contact its provider, the type of data provided by the service, and so forth. In order to allow interoperability between server and client software from multiple vendors, the request and response must be standardized. By focusing standardization on the interface, the software developers, data custodians, and processing-service providers can work to their particular software components, while connecting with other systems for complementary services. The following figure illustrates a basic OGC GIS service-oriented architecture.

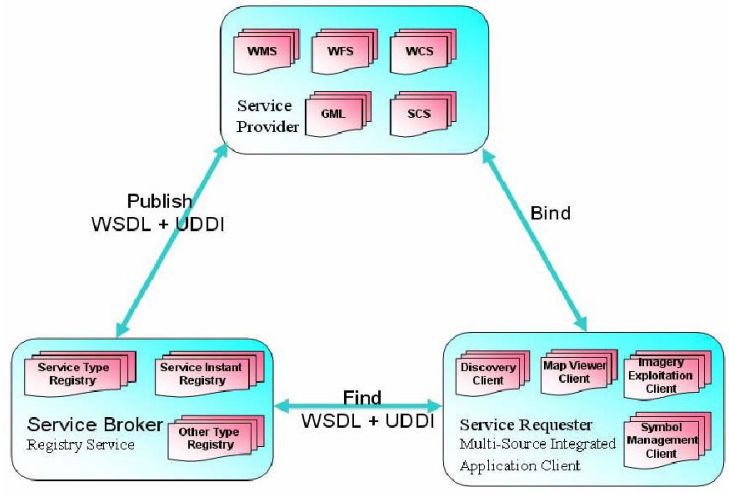

Figure 1. GIS Web services oriented architecture

Open GIS Consortium is developing specifications to standardize the message interfaces. These include: Web Map Service (WMS) for maps encoded as images; Web Feature Service (WFS) for geographic objects or vector data; Web Coverage Service (WCS) for continuous data; Sensor Collection Service (SCS) for live access to observations from sensors; Geography Markup Language (GML): XML components for encoding geographic objects for transport [12].

The OGC WMS specification offers a standard client-server interaction protocol that each map server implements as a common interface for accepting requests and returning responses. The same client is able to access to all available OGC web map servers over the Internet. The client through the common interface accesses each map server. In a distributed OGC WMS, a WMS server can also run as a WMS client that requests capabilities and maps from other WMS servers.

OGC WMS is an interoperable web mapping system. It provides common interfaces to connect with the client application and dynamically process geo-referenced data such as geographic maps and features coded using Geography Markup Language (GML) documents. The OGC Web Map Service specification defines a set of functions that clients may use to interact with WMS providers (servers). Any client making requests that conform to the specification can interact with any server that implements the WMS service. In effect, this creates an interoperable, distributed web mapping systems. An OGC web map server implements three functions: GetCapabilities, GetMap, and GetFeatureInfo. The GetCapabilities function provides the client with a map server's service metadata, specifying its capabilities. The
GetMap function specifies map request parameters that enable the client to request an image map. Finally, the GetFeatureInfo function allows the client to request more information about features at a specific location in the map.

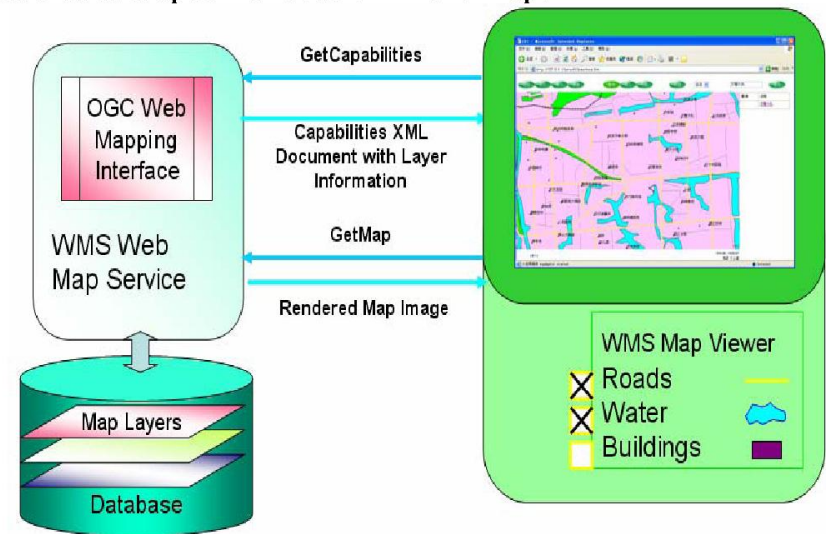

Figure 2. OGC WMS operation process for mapping application.

The OGC WMS operation process for mapping application is illustrated in Figure 2. The WMS GIS Web Service provides simple operational interface which can directly mapping to the Web service for operation. The client application requests the information from the web map server. The map server retrieves from the database the appropriate layers of geo-feature data for the specified spatial domain and generates a map that can be viewed directly in a graphical web browser or other pictorial software. All the business logic can be achieved by the exterior layer that data access layer can be directly connected to the customer applications.

III. Developing Web GIS Based Intelligent Transportation APPLICATION System With WEB SERVICE TECHNOLOGY

According to the OGC GIS Web Services framework, and integration SOA architecture, the multi-layers Web GIS Services system components can be constructed. The Web GIS based ITS application system provides a web GIS environment by the dynamic integrating GIS-T service components of vector and raster maps to customized ITS application system with geo-reference data and information. It supports the complex rendering, navigation and allows working with multiple layers, thematic maps, hyper linked features and attribute data. Based on the web service oriented architecture, the map features are stored on a SQL database according OGC SFS and WMS that can be used as raster maps source. The GIS data interface supports the ArcView shape files and MapInfo MIF files

The server side can be constructed based on J2EE architecture. The system architecture, system functions, and system security are the key technical problems should be investigated. The architecture of the GIS Web service can be logically divided into different layers with a particular service. Application layer includes the map check devices, customer's map divices, and sensors clients. Web application services layer includes application integration servers, soap servers, 
spatial information operation components, such as mobile positioning server and registration server components.

Once the GIS web services are construed, they can be used by a number of ITS application systems which include the intelligent transportation control systems, the video supervisory systems, the electronic police systems, the $110 / 122$ alarm systems, vehicle movement management systems, the street intersection control systems and the mass transportation systems. A customized ITS application system, which need the geo-reference data and information, can be viewed directly in a graphical web browser or other pictorial software. The ITS application system can integrated the GIS client and web map service by Hypertext Transfer Protocol. The GIS-T web service architecture includes the web service engine, ITS application clients and web service interface and data storages.

GIS-T Web Services have many advantages. GIS-T Web Services are discoverable, self-describing software components UDDI is an open standard with broad industry support standard. When an implemented GIS Web Service exposed in any Web Services portal, it can be discovered in any Web Services portal. Once a GIS Web Service is discovered, the developer can begin using it immediately. All they need is the full URL path to the services WSDL. Each method, parameter, property, and return value of the service is described in a standard way, allowing modern development tools to access to the exposed functionality.

GIS-T Web Services are easily implemented compare to the traditional GIS system. GIS-T Web Services conceal complexity. Web Services embed its complex data processing within itself in server side. Client side application developer make use of Web Services through standard interface which described in Web Services WSDL, therefore the application developer don't need dealing with Web Services internal process.

The following basic GIS-T Web Service should be considered in the design for the service oriented distributed GIS-T platform. They provide a variety of interfaces for integration and interoperability with customized ITS application system.

GIS-T Web services: Web GIS-T platform should support the standard enterprise application server technologies to build web services with embedded d spatial services, software components and APIs to easily embed spatial capabilities in customized ITS application system built using standard technologies and architectures.

Web Mapping Services (WMS): WMS can display map layers for all the customized ITS application system and allow all the customized ITS application system to display map layers from Web GIS platform

OpenGIS Consortium Interoperability Services and Standards: The Web GIS platform is implemented with the OpenGIS interoperability standards:

Geography Markup Language (GML): GML is a common XML-based file format for exchanging spatial information and attributes. The Web GIS platform should support the GML standards.

The GIS-T platform architecture is designed as a multi-layer architecture that integrated the web service, Servlet/JSP functions and GIS APIs based on the framework of J2EE infrastructure. It is a distributed, platform independent system architecture. The data are stored and managed with EJB. The distributed systems enable the databases and services in the same or different computers.

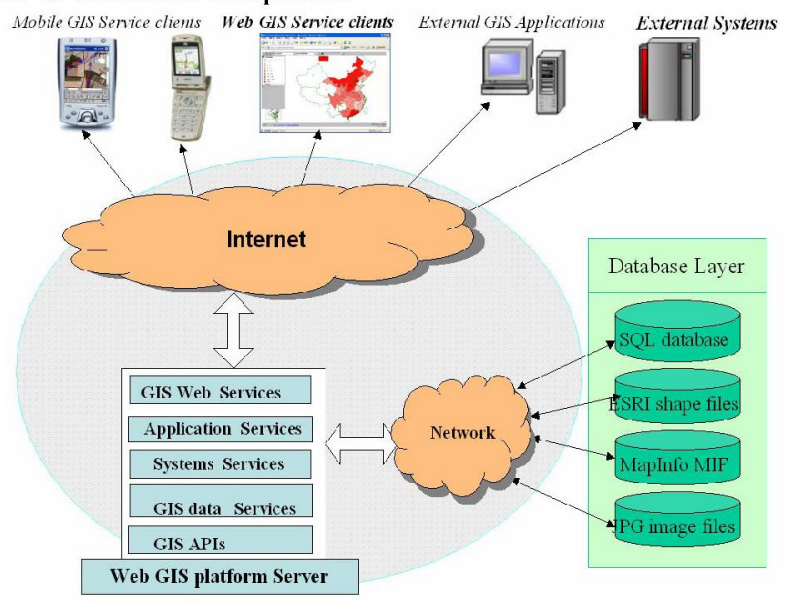

Figure 3. Web GIS-T platform server environment.

The Web GIS platform Server architecture is consisted of three layers: User Interface Layer, Application Server Layer and Database Layer. The figure 3 shows the Service oriented architecture of GIS-T platform.

Application Server Layer: In the application server layer, the GIS web services, Servlet/JSP interface, GCO services that include GML and WMS map service, and basic system application service deployed in the J2EE server container. The GIS function such as the zooming out and in, viewing, panning and finding be designed as a function session bean that can be accessed by the GIS web service, Servlet/JSP and other Application interfaces. The application server layer is built as a J2EE application, with several EJB modules, to provide servercentric spatial functions to applications. Key components of the server that implemented as EJBs are Web GIS services, Geo-spatial functions, such as the viewing, layer selection, editing, querying, and analysis Data Source Management.

Database Layer: The GIS data are stored and managed with the distributed systems architecture that enables the GIS data, databases and services in the same or different computers. The OpenGIS Consortium has provided specifications on spatial entities and the space / time reference systems.

User Interface: Web GIS platform provides a number of user interface components: Web service client, GIS java Applet and any other web service enabled applications. The mobile and PDA that support the web service standards interface could get the map and geo-referenced data through the GIS web service interface. A GIS Java Applet is another user interface that can be used to retrieve and handle the vector and raster map using the map tools. Java applet enables spatial 
viewing, querying, and richer editing capabilities to be easily embedded in desktop and web applications.

\section{IMPLEMENTATION AND FURTHER WORK}

According to the service-oriented architecture proposed in above section, we designed and implemented a prototype of the service enabled Web-GIS intelligent transportation application system. Figure 5 shows the screenshot of our system. It is a distributed, platform independent system architecture that be accessed by lots of different computers in network with different kinds of operating system. The web service framework is applied into the GIS system design. GIS web services can provide hosted spatial data and GIS functionality and integrate to the customized ITS application system to perform basic geo-processing tasks, such as address matching, map image display, and routing, without maintaining GIS tools or the associated geographical data.

The system provides an efficient means for the transportation planning with following advantages. Firstly, the hosted spatial data and GIS functionalities that can be accessed and integrated to the different customized GIS-T applications to meet the practical need in the processes of transportation planning. Secondly, running on the Internet, the system is accessible from anywhere in the world. Web-GIS service technologies not only make the system broadly accessible through Internet and but also provide the precise georeferenced data for public and transportation planning researchers.

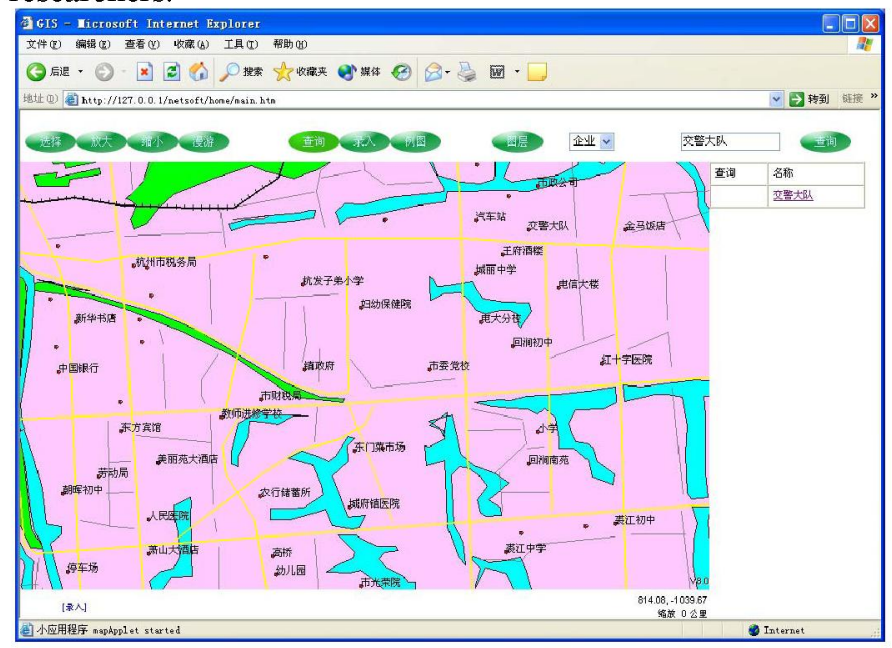

Figure 4. GIS-T Web service used in the transportation planning information.

Because the theory and application of GIS Web Service technology in the continual development, the GIS-T Web Service still leaves many the problem that needed to be investigated. Our research work will be focus on the following problems: (1) Since the requirement of the practical GIS applications in different application domain and field are quite complicated, a general GIS-T Web service should be integrated into the different user customized application. (2) GIS spatial analysis web service is needed for historical data and real-time data. GIS spatial analysis service plays in important role in the ITS application systems. For example, for the problem of choosing the best traffic routes, the GIS spatial analysis service is urgent needed. (3) Space-Time GIS-T web service refers the GIS historic data charging. When the city roads are constructed continuously, the new urban road network and related data in GIS-T service needed updating. On the other hand, the realtime traffic flow data, traffic control data, and GIS-T GPS data are also in continuous changing. How to manage and dealt with the space-time issue is a very important research subject.

\section{CONCLUSION}

As the new generation GIS technology, the GIS-T Web Services are development-oriented, standards-based core technologies that have broad prospects for development of Web GIS based intelligent transpiration application systems for different specific transportation information systems. The GIS$\mathrm{T}$ web services provide the hosted spatial data and GIS functionality to integrate the customized ITS applications to perform basic geo-processing tasks, such as address matching, map image display, and routing, without maintaining GIS tools or the associated geographical data. Through GIS-T web service, the hosted spatial data and GIS functionalities that can be accessed and integrated to the different customized ITS applications. The service-oriented architecture is a very promising architecture for practical implementation of the next generation GIS-T information systems. It has good promotion prospects and the important reference value for the system design and construction of Web GIS ITS application systems.

\section{REFERENCES}

[1] Gui Lan, Li Yaojun, Chen Xinfa, "Study and Development of WebGIST for Transportation", Journal of Changsha Communications University, Vol.17 No.4,pp.18-22, 2001.

[2] Gil, Nicholas T. and Al-Akhras, Ahmad, "Transportation Plan Information Management System", Proceedings of the Conference on Transportation, Land Use, and Air Quality May, pp. 606 - 613. 1998.

[3] Winnie Tang and Jan Selwood, "Connecting Our World: GIS Web Services", ESRI Press, 2003.

[4] IBM, "Web Services architecture overview", Available at http://www106.ibm.com/developerworks/ webservices/library/w-ovr/. 2003

[5] Domenico Cotroneo, Cristiano di Flora and Stefano Russo, "An Enhanced Service Oriented Architecture for Developing Web-based Applications" , J. Web Eng., 1(2), pp. 128 146, 2003.

[6] Xuefeng Li, Shengru Tu, Xiangfeng He, Jay J. Ratcliff, "Web-Based Distribution of GIS Metropolitan Maps", Fifth International Conference on Information Visualization (IV'01) London, England, pp.419-424, July, 2001

[7] Kingston, R., Carver, S., Evans, A. and Turton, I, "Web-Based Public Participation Geographical Information Systems: An Aid To Local Environmental Decision-Making", Computers, Environment and Urban Systems, Vol. 24, No. 2. pp. 109-125, 2000.

[8] ESRI, "An Overview of ArcWeb Services", Available at http://www.esri.com/library/whitepapers/pdfs/arcweb-services.pdf. July 2004.

[9] Mircosoft, "MapPoint .NET Demos," Available at http://demo.mappoint.net/. 2003.

[10] Open GIS Consortium Ine, "OpenGIS Simple Feature Specification For SQL Version 1.1. Open GIS project document 99-049", May 1999.

[11] Open GIS Consortium Inc, "Web Map Service Implementation Specification 1.1.0. Open GIS project document: OGC 01-047r2", June 2001.

[12] Cox, S., Daisey, P., Lake, R., Portele, C. and Whiteside, "A. Geography Markup Language (GML) Implementation Specification version 3.0.0: OpenGIS project document: OGC 02-023r4", Available at http://www.opengis.org/techno/ documents/02-023r4.pdf. 2002. 